\title{
In-Vitro Platform to study Ultrasound as Source for Wireless Energy Transfer and Communication for Implanted Medical Devices
}

\author{
Francesco Mazzilli, Michela Peisino, Rostand Mitouassiwou, Benjamin Cotté, Prakash Thoppay, \\ Cyril Lafon, Patrick Favre, Eric Meurville, and Catherine Dehollain
}

\begin{abstract}
A platform to study ultrasound as a source for wireless energy transfer and communication for implanted medical devices is described. A tank is used as a container for a pair of electroacoustic transducers, where a control unit is fixed to one wall of the tank and a transponder can be manually moved in three axes and rotate using a mechanical system. The tank is filled with water to allow acoustic energy and data transfer, and the system is optimized to avoid parasitic effects due to cables, reflection paths and cross talk problems. A printed circuit board is developed to test energy scavenging such that enough acoustic intensity is generated by the control unit to recharge a battery loaded to the transponder. In the same manner, a second printed circuit board is fabricated to study transmission of information through acoustic waves.
\end{abstract}

Index Terms-biomedical telemetry, energy scavenging, implanted medical devices, ultrasound, sensor networks, wireless communication.

\section{INTRODUCTION}

In the 1950s ultrasound started to be used for biomedical imaging and for therapy purposes [1] and a recent work has shown the ability of acoustic waves to energize and transmit information within the human body [2]. By exploiting the capability of acoustic waves to penetrate deeper in the body tissue without being significantly attenuated [3], [4], new implanted medical devices (IMDs) are appearing among health monitoring applications [5].

One important requirement for IMDs is longevity, which is determined by the energy storage capabilities [6], and by the modulator power consumption within the transponder. Therefore, a load modulation technique (backscattering modulation), largely exploited in radio frequency identification (RFID), may be the best candidate for medical devices due to its low power consumption [7]. However, IMDs should be tested in-vitro for

F. Mazzilli, P. Thoppay and C. Dehollain are with the RF IC Group at the Ecole Polytechnique Fédérale de Lausanne (EPFL), 1015 Lausanne, Switzerland. (Email: francesco.mazzilli@epfl.ch).

M. Peisino and E. Meurville are with the Laboratory of Microengineering for Manufacturing, Ecole Polytechnique Fédérale de Lausanne (EPFL), 1015 Lausanne, Switzerland. (Email: michela.peisino@epfl.ch).

R. Mitouassiwou and P. Favre are with the Institute for Information and Communication Technologies, Haute Ecole d'Ingénierie et de Gestion du Canton de Vaud, 1401 Yverdon, Switzerland.

B. Cotté and C. Lafon are with the Institut National de la Santé et de la Recherche Médicale (INSERM), U556, 69424 Lyon, France. primary analysis of side effects so that this new technology can be accepted for clinical use.

The paper discusses the possibility of using acoustic waves to charge a lithium battery and to allow wireless communication in IMD applications. In Section II motivation of the work is presented focusing on medical applications. In Section III an in-vitro platform to characterize ultrasound is described and in Section IV the construction of the platform is presented. In Section $\mathrm{V}$ experimental results of the use of ultrasound as source for wireless energy transfer and communication are shown. Lastly, in Section VI the conclusion is presented.

\section{Motivation}

A continuous monitoring system such as an IMD with processing capability and able to communicate wirelessly could help a patient suffering from chronic diseases. Heart rhythm abnormalities such as atrial fibrillation are commonly encountered in clinical practice and regular monitoring is required to ensure control of the heart rate. Hypertension is another cardiovascular disease and an intelligent IMD, such as the proposed ultrasonic transponder system, would enable physicians to monitor patients with high blood pressure during normal activity. Also elderly patients, a group whose life expectancy is continuously increasing can benefit of the ultrasonic transponder system. In this case identifying ways of surveying in a minimally invasive manner in their domestic environment is very important especially during months of non-temperate weather (either very cold or very hot). This would allow earlier detection of any degradation in their condition.

\section{SySTEM DESCRIPTION}

Fig. 1 shows the platform block diagram, where the control unit (CU) and the transponder (TR) transducers are represented by an equivalent electrical impedance $Z_{C U}$ and $Z_{T R}$, respectively. The acoustic waves are sent from the $\mathrm{CU}$ to the TR covering a distance $\mathrm{d}$ at the operating frequency $f_{0}$. A source signal, $V_{I N}$, is applied to the $\mathrm{CU}$ that generates mechanical vibrations in the direction of the TR, the output signal produced corresponds to the available voltage of the implanted circuit, $V_{A V}$. Following, $V_{A V}$ is boosted up through 
a shunt inductor, $\mathrm{Lp}$, and rectified using an $\mathrm{AC}$ to $\mathrm{DC}$ converter. The rectified voltage, $V_{R E C}$, has to be set at the correct constant level to recharge the battery, within the micro power module, while keeping the delivered current, $i_{I N}$, high enough to obtain a quick charge. Once, the energy storage element is completely charged, the load is connected to the battery that delivers the current $i_{O U T}$. This is the energy harvesting chain named as block A, a second block B represents the wireless communication system. A simple modulator mechanism was developed to transmit a coded signal from the TR to the CU, which contains the sensor information stored in the randomaccess memory (RAM), through a microprocessor.

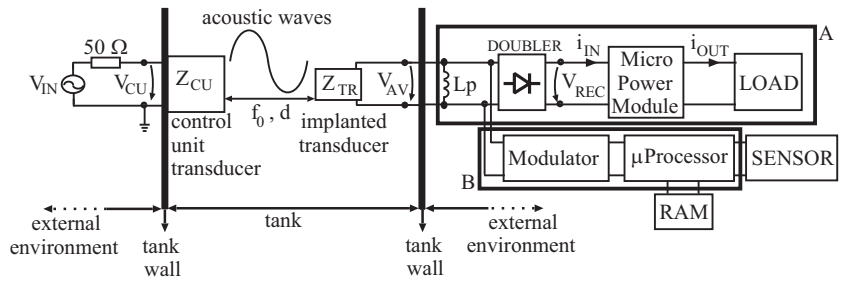

Fig. 1. Block Diagram of the Platform.

Fig. 2 shows the complete schematic of the energy harvesting circuit, where the battery is recharged through acoustic waves. The differential output of the implanted transducer, $V_{A V+}$ and $V_{A V-}$, is fed into a doubler circuit which yields an output signal equal to:

$$
V_{R E C}=2 \hat{V}_{A V+}-2 V_{D}
$$

Where $\hat{V}_{A V+}$ is the positive peak amplitude of the implanted transducer, $V_{D}$ is the threshold voltage of diodes D1, D2 and $V_{A V-}$ is connected to the ground plane. The voltage at node $\mathrm{A}$ is set to $4.1 \mathrm{~V}$ by the reference diode. This $4.1 \mathrm{~V}$ is the voltage needed to recharge the battery when transistor Q1 is forward biased. So, the rectified voltage, $V_{R E C}$, across the output capacitor, $C_{O U T}$, has to be at least $4.7 \mathrm{~V}$. Considering that the receiving area is quite small, this minimum required voltage is hard to achieve while keeping an excitation voltage, $V_{C U}$, low enough to prevent hot spots in the IMD. Therefore, $V_{R E C}$ can be boosted up by three times by adding a high-Q parallel inductor $L p$ at the input of the doubler. Lp cancels out the effect of parasitics due to the transducer and the imaginary part of the input impedance of the rectifier. Also two protection circuits are used to prevent reverse current flowing toward the input and to avoid deep discharge of the battery by the load, $R_{L O A D}$

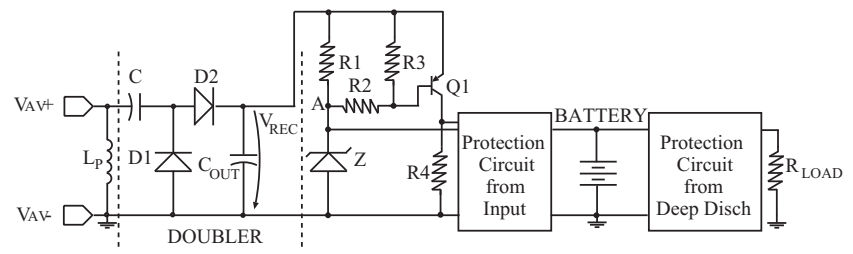

Fig. 2. Schematic of the front end energy harvesting circuit.

Fig. 3 shows an amplitude modulation (AM) technique known as backscattering modulation and also called echo modulation. The load seen by the implanted transducer affects the reflection or the absorption of the transmitted signal from the CU towards the TR. A $\mu$ processor drives both switches $\mathrm{S} 1$ and $\mathrm{S} 2$ through a control signal, $V_{c t r l}$. If $V_{c t r l}=\operatorname{logic}$ 1 , then $\mathrm{S} 1$ and $\mathrm{S} 2$ connect the capacitor $\mathrm{C}$ to $Z_{T R}$ making the transducer stiffer and reflecting back the incoming signal so a high state is transmitted to the $\mathrm{CU}$ receiver. When $V_{c t r l}=\operatorname{logic}-0$, then $\mathrm{S} 1$ and $\mathrm{S} 2$ short circuit $\mathrm{C}$ to ground and the transducer is able to vibrate allowing the incoming signal to be absorbed, thus transmitting back a low state.

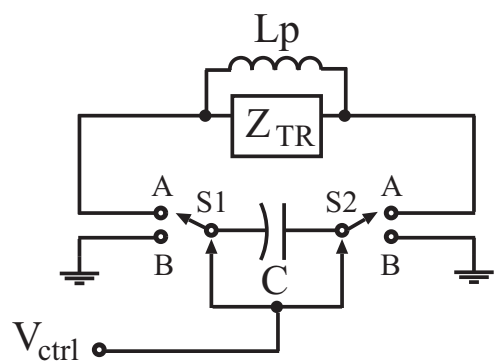

Fig. 3. Modulation technique: when switches $\mathrm{S} 1$ and $\mathrm{S} 2$ are in position $\mathrm{A}$, a capacitance $\mathrm{C}$ prevents the transducer from vibrating and this results in signal reflection, thus transmitting a high state value; when switches S1 and S2 are in position $\mathrm{B}$, the inductance $\mathrm{Lp}$ increases the magnitude of the transducer impedance, which results in signal absorption and so a low state value is transmitted.

\section{Construction of the Platform}

Fig. 4 shows the in-vitro platform used to hold a pair of acoustic transducers, either single elements or an array, placed inside a $50 \times 50 \times 50 \mathrm{~cm}^{3}$ plexiglas tank. Fig. 4(a) presents the pair of transducers used for the energization test made by Ferroperm Piezoceramics A/S. The CU transducer (Ferroperm Pz28) is a single element focused transducer having the diameter of $50 \mathrm{~mm}$ and a radius of curvature of $50 \mathrm{~mm}$. The $\mathrm{CU}$ transducer is attached to one of the walls of the tank while the transponder transducer (Ferroperm Pz26), represented by a piston having a diameter of $6.35 \mathrm{~mm}$, is held by a rod at a distance of $50 \mathrm{~mm}$ at which the maximum transfer of energy occurs. To test the acoustic wireless communication another pair of transducers are used which are made by IMASONIC, a $1 \mathrm{MHz}$ flat array (64 elements) used as $\mathrm{CU}$ transducer and a $1 \mathrm{MHz}$ flat single element transducer used for the transponder. Each element of the CU transducer has an area of $30 \times 1.55 \mathrm{~mm}^{2}$, the pitch between each element is $2.05 \mathrm{~mm}$ and the whole transducer bandwidth at $-6 \mathrm{~dB}$ is $65 \%$. The transponder transducer presents a bandwidth of $60 \%$ at $-6 \mathrm{~dB}$, and a diameter of $13 \mathrm{~mm}$.

In order to reduce standing waves and echoes, an absorber material made of silicone is placed all around the two transducers (Aptflex F28) [8]. In Fig. 4(a) the absorber material is only present behind the CU transducer in order to clearly show the TR transducer. Fig. 4(b) shows the mechanical system used to move the piston transducer inside the tank along the $\mathrm{x}, \mathrm{y}$ and $\mathrm{z}$ axes with a resolution of $50 \mu \mathrm{m}$, also having the possibility to be rotated $\pm 25^{\circ}$ along the $\mathrm{x}$ axis. 


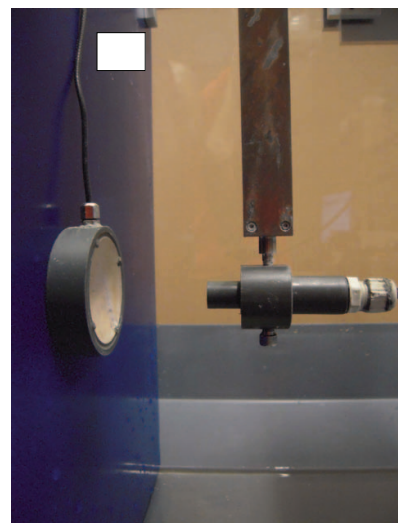

(a)

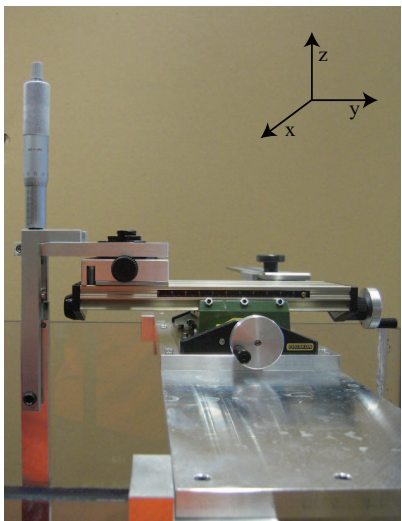

(b)
Fig. 4. Photograph of the in-vitro platform. (a) The pair of transducers used for the test, CU attached to the absorber material ( $\square)$, (b) mechanical system to move the TR along the $\mathrm{x}, \mathrm{y}$ and $\mathrm{z}$ directions.

\section{Measurements}

Two different PCBs were fabricated to evaluate the ability of acoustic waves to recharge a lithium battery with a capacity of $300 \mu A h$, manufactured by Infinity Power Solution [9], and also to transmit information.

\section{A. Wireless Energy Transfer}

The maximum derated spatial-peak temporal-average acoustic intensity allowed by the Food and Drug Administration (FDA) is $I_{S P T A, 3}=430 \mathrm{~mW} / \mathrm{cm}^{2}$ for cardiac applications [10]; therefore, considering the TR piston transducer in Fig. 4(a) at a distance $d$ from the $\mathrm{CU}$, where the maximum of the transmitted acoustic intensity is detected, the peak amplitude of the received available voltage can be defined as:

$$
\hat{V}_{A V} \leq \sqrt{2 \eta_{T R} A_{T R} I_{S P T A, 3} \frac{\left|Z_{T R}\right|}{\cos \left(\varphi_{T R}\right)}}
$$

Where the TR transducer is characterized by an aperture area $A_{T R}=31.6 \times 10^{-2} \mathrm{~cm}^{2}$ and an electro-acoustic efficiency $\eta_{T R}=10 \%$; at $f_{0}=1.033 \mathrm{MHz}$ the equivalent impedance modulus and phase are measured to be $\left|Z_{T R}\right|=700 \Omega$ and $\varphi_{T R}=-58^{\circ}$, respectively. Moreover, $V_{R E C}$ maximum is derived by substituting (2) in (1).

Fig. 5 shows the current to the battery sinks during charging and the voltage level across the battery. It is clearly visible that as the $V_{C U}$ increases, $V_{R E C}$ increases and the recharging time decreases. Moreover, the battery is defined as charged when the voltage across its pins is equal to $4.1 \mathrm{~V}$. The status of the battery charge, both current and voltage, is monitored by a voltmeter and an amperometer through a LabVIEW program installed on a PC.

Fig. 6 presents the voltage discharging profile versus time for different resistive loads. The battery is disconnected from the load when the voltage drops down to $2.5 \mathrm{~V}$ by the deep discharge protection circuit to prevent the battery from being damaged. In Fig. 6(b) for a $R_{L O A D}=5.6 \mathrm{k} \Omega$, the battery is out of charge after 20 minutes. In case of IMDs, the load

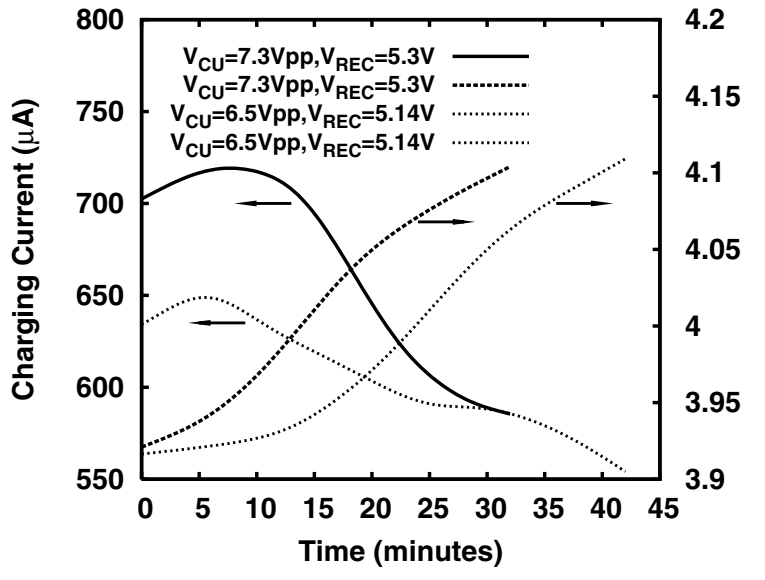

Fig. 5. Charging current and voltage profile.

is a sensor and it sinks current only for few seconds and then it is turned off by a $\mu$ processor. Therefore, assuming a fully charged battery, $4.1 \mathrm{~V}$, and a load of $5.6 \mathrm{k} \Omega$, the delivered current is $i_{O U T}=732 \mu \mathrm{A}$. Moreover, considering an operation of the load for only $200 \mathrm{~ms}$ per minute, the battery lasts for 4 days.

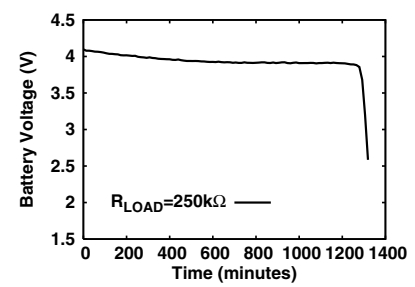

(a)

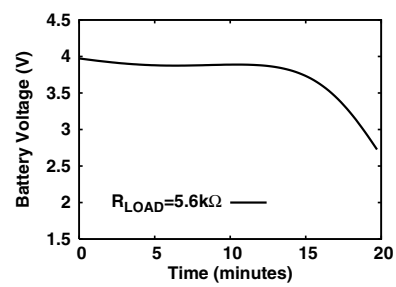

(b)
Fig. 6. Discharging voltage profile for different loads: (a) $R_{L O A D}=$ $250 \mathrm{k} \Omega$, (b) $R_{L O A D}=5.6 \mathrm{k} \Omega$.

\section{B. Wireless Communication}

Fig. 7 presents the control unit (CU) and transponder (TR) within the acoustic communication subsystem. To prove the wireless data transmission, a CU transducer made of sixty-four elements is used, where only one element is used to transmit an acoustic signal, $Z_{C U, T X}$, and an adjacent element, $Z_{C U, R X}$, is used to detect the reflected wave yielded from the TR.

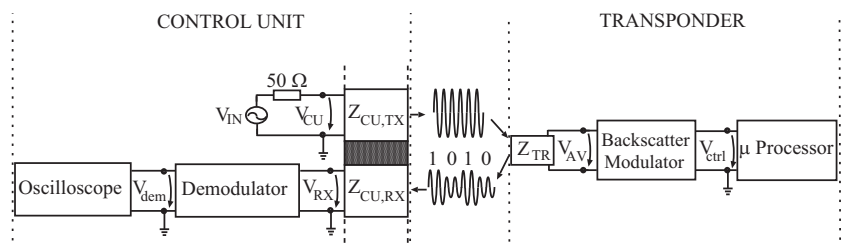

Fig. 7. System communication block diagram.

Fig. 8 shows the signal used to excite $Z_{C U, T X}$ and the received signal $V_{A V}$. A message is stored in the $\mu$ processor and used as control signal, $V_{c t r l}$, by the backscatter modulator 
(Fig. 3). Fig. 9 presents $V_{c t r l}$ and the demodulated signal, $V_{d e m}$. The fact that $V_{d e m}$ is represented by a square wave is due to the presence of a cascade by the two amplifiers in the receiver demodulator block; a single stage amplifier has a gain of $40 \mathrm{~dB}$ so that the input sinusoidal signal, $V_{R X}$, saturates.

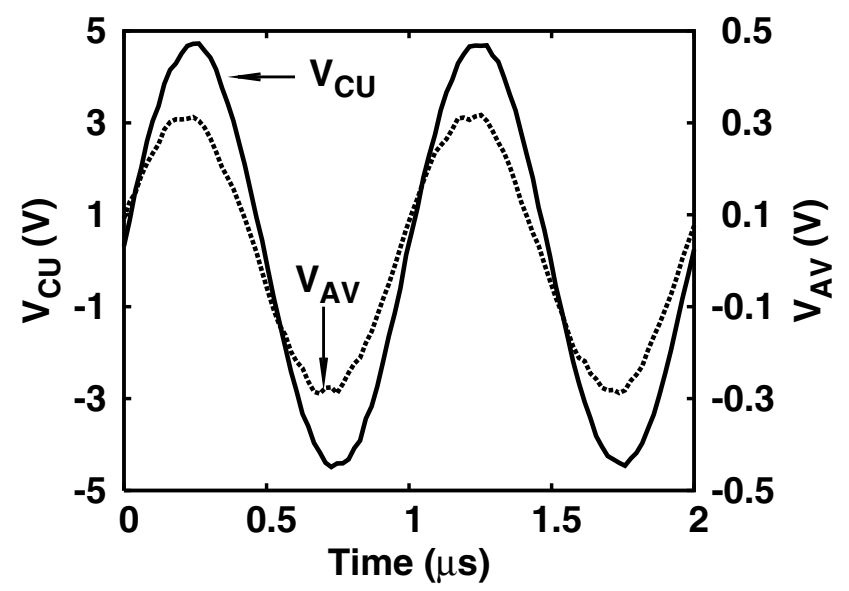

Fig. 8. Transmitted signal from the control unit and received signal to the transponder.

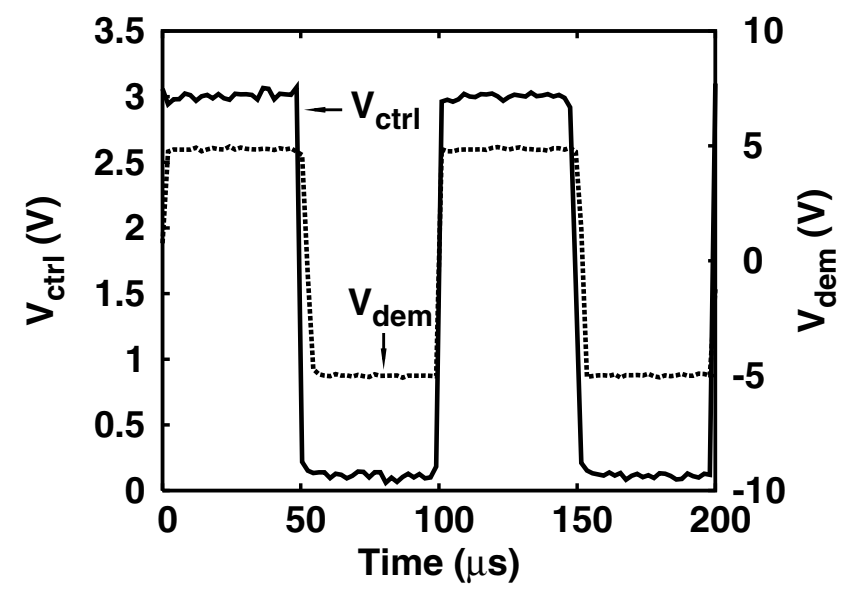

Fig. 9. Message transmitted from the transponder to the control unit through backscattering and demodulated signal generated by the control unit.

In the demodulator it is preferable to use a comparator with an adjustable threshold according to the minima and maxima of the received signal, $V_{R X}$, in order to get rid of the crosstalk between $Z_{C U, T X}$ and $Z_{C U, R X}$. Moreover, the transponder system should be autonomous when wireless communication occurs so during testing it is necessary to avoid any external power supply henceforth a battery has to be embedded.

\section{CONCLUSION}

An in-vitro platform to study ultrasound as source for wireless energy transfer and communication has been proposed. A system description of the platform, including the mechanical system to hold and move a control unit and a transponder, was given. Two PCBs were fabricated in order to be able to recharge a lithium battery and to implement a backscatter modulator. Henceforth, this set-up allows the engineer to design an in-vitro testing tool for in body acoustic wave propagation and to solve communication issues such as interference that may occur among elements of the same array, which is referred as cross-talk.

\section{ACKNOWLEDGMENT}

The research leading to these results has received funding from the European Community's Seventh Framework Programme (FP7/2007-2013) under grant agreement n. 224009. The authors thank Giancarlo Corradini (Laboratory of Microengineering for Manufacturing, EPFL), who has helped in the construction of the platform, and IMASONIC, that has provided the transducers to test the wireless communication.

\section{REFERENCES}

[1] J. Aldes, W. Jadeson, and S. Grabinski. A new approach to the treatment of subdeltoid bursitis. American journal of physical medicine, 33:79-88, 1954.

[2] Hideyuki Kawanabe, Tamotsu Katane, Hideo Saotome, Osami Saito, and Kazuhito Kobayashi. Power and information transmission to implanted medical device using ultrasonic. Japanese Journal of Applied Physics, 40(Part 1, No. 5B):3865-3866, 2001

[3] Tomohiro Yamada, Takumi Uezono, Kenichi Okada, Kazuya Masu, Akio Oki, and Yasuhiro Horiike. Rf attenuation characteristics for In Vivo wireless healthcare chip. Japanese Journal of Applied Physics, 44(7A):5275-5277, 2005.

[4] Antonis Ifantis and Antonis Kalis. On the use of ultrasonic communications in biosensor networks. In BIBE, pages 1-6, 2008.

[5] Binh C. Tran, Bin Mi, and Robert S. Harguth. Systems and methods for controlling wireless signal transfers between ultrasound-enabled medical devices. patent, (20090201148A1), 2009.

[6] Xiaojuan Wei and Jing Liu. Power sources and electrical recharging strategies for implantable medical devices. Frontiers of Energy and Power Engineering in China, 2:1-13, 2008.

[7] J.-P. Curty, N. Joehl, C. Dehollain, and M.J. Declercq. Remotely powered addressable uhf rfid integrated system. Solid-State Circuits, IEEE Journal of, 40(11):2193-2202, Nov. 2005.

[8] http://www.acoustics.co.uk/products/aptflex-f28.

[9] Infinity Power Solutions. Standard product selection guide.

[10] Food and Drug Administration. Information for Manufacturers Seeking Marketing Clearance of Diagnostic Ultrasound Systems and Transducers, 2008. 